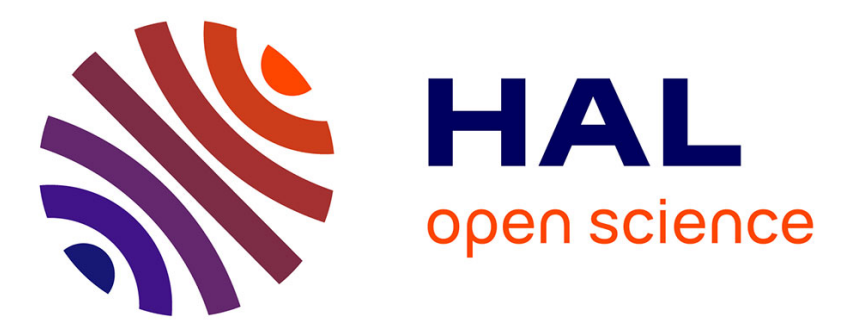

\title{
Doris Lessing, la force obscure des femmes
}

Françoise Hatchuel

\section{To cite this version:}

Françoise Hatchuel. Doris Lessing, la force obscure des femmes. Revue Esprit, 2007, pp.238-241. 10.3917/espri.0712.0238 . halshs-00354107

\section{HAL Id: halshs-00354107 https://shs.hal.science/halshs-00354107}

Submitted on 13 Dec 2017

HAL is a multi-disciplinary open access archive for the deposit and dissemination of scientific research documents, whether they are published or not. The documents may come from teaching and research institutions in France or abroad, or from public or private research centers.
L'archive ouverte pluridisciplinaire HAL, est destinée au dépôt et à la diffusion de documents scientifiques de niveau recherche, publiés ou non, émanant des établissements d'enseignement et de recherche français ou étrangers, des laboratoires publics ou privés. 


\section{DORIS LESSING, LA FORCE OBSCURE DES FEMMES}

"Icône du féminisme ", " prix Nobel prix rebelle " "écrivaine engagée " : les malentendus sont nombreux à propos de Doris Lessing, et elle est la première à les relever, de cette ironie lucide qui la caractérise. Ne retenir de Doris Lessing que des combats politiques " extérieurs ", c'est oublier les images profondes qui traversent l'ensemble de ses écrits et tissent, d'œuvre en œuvre, tout un réseau de chambres (j'y reviendrai), de corres- pondances et de combats non pas politiques et organisés mais vitaux et sensoriels. Dans une approche lucide mais non culpabilisante, elle décrit, inlassablement, et ne prescrit jamais. Elle dit ce qui est, implacablement, jamais ce qui devrait être, et que chacun et chacune s'en débrouille ensuite. Descriptions magistrales du difficile travail de vivre tel que l'accomplissent des personnages aussi variés qu'un jeune noir analphabète ( La faim» dans la Madone noire, deuxième tome des Nouvelles africaines ${ }^{1}$ ), un quatuor vivant une histoire d'amour hors norme ${ }^{2}$, voire deux insectes attelés à transporter une infime et précieuse particule de bouse de vache ${ }^{3}$.

C'est pourquoi Doris Lessing n'est pas «provocatrice» ou "rebelle», dans la dérive que prennent trop souvent ces termes, où il s'agirait de dénoncer, contester et préconiser.

Doris Lessing étonne par sa puissance d'évocation et son acuité d'analyse de la condition humaine. Non pas, à l'égard par exemple d'un Malraux, une condition humaine dans ses dimensions conscientes et rationnelles (quels choix devons-nous faire, comment rester fidèle à ses idéaux, que décider face au danger, etc.) mais dans un registre beaucoup plus souterrain du non-dit et de l'intime, où l'angoisse et la dislocation sont non plus des artéfacts à écarter mais le cœur même de la vie. À l'image de Toni Morrisson ou Elfriede Jelinek, deux autres grandes Nobel de l'expérience féminine, Doris Lessing excelle à montrer (il faudrait même dire "à mettre en sens ") la densité des tâches qui incombent aux

1. Doris Lessing, Nouvelles africaines, t. II : la Madone noire, Paris, La Découverte, coll. " La Découverte-poche ", 1988 (éd. originale 1954). 2006 .

2. Id., les Grands-mères, Paris, Flammarion,

3. Id., "Le soleil entre les pattes ", dans la Madone noire, op. cit. 
femmes et la force souterraine qu'il leur faut pour les affronter, faire face à la réalité et l'assumer.

Dans les Enfants de la violence ${ }^{4}$, elle montre pourquoi naître en 1919 , de parents qui ont connu toute l'horreur de la guerre (sa mère était infirmière, son père fut amputé d'une jambe) n'avait rien d'anodin, et comment la violence est transmise et traverse nos vies, qu'on le veuille ou non. Non seulement la violence physique des coups ou de la maltraitance, mais bien celle, sourde et non moins pernicieuse, des réseaux d'obligations et d'injonctions qui emprisonnent et terrorisent. Saisissantes scènes d'emprisonnement psychique, notamment à l'égard des enfants, mais aussi bien sûr des femmes, blancheur chirurgicale, traque de la saleté (accentuée par l'expérience fondatrice qu'a connue Doris Lessing, confrontée à une mère attachée à transformer la brousse rhodésienne en parfaite réplique de l'Angleterre), plainte de la mère qui projette sur son enfant son découragement à résister à la lente déroute, "regards de propriétaire 5 ", petite fille "cramponnée aux bras, se protégeant de l'orage de critiques et d'injures ${ }^{6}$ ", etc. Nul-le n'a, mieux qu'elle, dit les pièges inévitables et les nécessaires faux-semblants, ni mis en scène, y compris de façon fantasmatique, le chaos de la vie.

Son œurre montre aussi que dans ce combat inégal, les femmes sont en première ligne, parce que c'est à elles qu'incombe l'accueil des enfants, et que toutes les sociétés ont dénié la difficulté que cela représentait, car l'affronter consciemment serait trop difficile. Les femmes sont ainsi renvoyées

4. D. Lessing, les Enfants de la violence, Paris, La Découverte, coll. «La Découvertepoche », 1979 (éd. originale 1951-1959).

5. Ibid.

6. Id., Mémoires d'une survivante, Paris, Albin Michel, 1982 (éd. originale 1974, p. 80). à une supposée incapacité personnelle contre laquelle elles n'ont plus qu'à faire face si elles ne veulent pas sombrer, s'organisant alors en modalités lourdement défensives qui feront peser un poids non négligeable sur les enfants. Nul-le mieux qu'elle n'a décrit le risque permanent de la dislocation et l'impossible, et pourtant nécessaire, rassemblement. C'est en montrant, au plus près, comment ses héroïnes s'en débrouillent, de façon forcément imparfaite, qu'elle nous aide à le faire. Elle rend quasiment palpable la façon dont nous nous accrochons aux bribes du monde que nous connaissons, ou croyons connaître, même lorsque celuici se délite.

Car si la surface, l'histoire apparente, changent en profondeur, ce sont les mêmes thèmes qui travaillent, et c'est dans ce sens qu'il s'agit bien là d'une œuvre, et non d'une succession de bons romans. De livre en livre se retrouve souvent un personnage de femme en lutte contre la déliquescence, qui range, trie, repeint, nettoie, jette, organise. Ainsi, la Terroriste ne raconte pas simplement l'" histoire de la dérive suicidaire d'un groupe d'apprentis révolutionnaires " selon une formule un peu passe-partout ${ }^{7}$ mais surtout une sombre affaire de maison vide et de rideaux enlevés, nettoyés, remis, transportés, perdus, retrouvés, et la façon dont ce symbole d'une vie « installée " fait écho à l'incapacité de l'héroïne à placer son énergie au bon endroit et à endiguer le virage que prend sa vie. La dérive n'est pas inexorable mais, à partir d'un certain moment, elle devient inéluctable, et nul-le ne peut dire pourquoi. Les choses adviennent, et le seul combat que l'on puisse mener consiste à faire avec, sans que cela ne dise en aucune façon comment on va le faire. L'héroïne

7. Le Monde du 12 octobre 2007. 
se bat, mais pas au bon endroit, et ce qui frappe peut-être le plus c'est l'étroitesse de la limite entre savoir et ne pas savoir, faire ce qui s'avérera par la suite pertinent et se tromper. Ses héroïnes ne proclament pas ce qu'elles vont faire. Elles sont prises dans des situations, et assument comme elles peuvent ce que, dans ces situations, elles sont en train de faire, en tâchant de rester au plus proche d'ellesmêmes, même quand elles se trompent. Sans excuse, sans justification, sans provocation et sans scandale. Du féminisme en actes, pas en mots, qui fait écho à l'inlassable travail psychique au lieu de le refouler et les met à l'opposé de ces "petits hommes " qu'elle dénonce dans son entretien au Monde des livres du 28 septembre 2007.

Dans ce travail de mise en scène de ce que demande le difficile travail de rassemblement, une place significative est donnée aux maisons et à la disposition des pièces. On se prend à rêver de ce qu'aurait inventé une Doris Lessing architecte ! Maisons collectives ou voisines, images de Martha Quest, I'héroïne de la saga autobiographique des Enfants de la violence s'efforçant de maintenir séparées les différentes "pièces " de sa vie, appartement en sous-sol qu'occupe, dans la suite de la saga, la maîtresse de maison de retour d'une hospitalisation psychiatrique, alors qu'une autre prend sa place " en haut ", notamment pour tenter de veiller sur son fils, pièces " de l'autre côté du mur » dans Mémoires d'une survivante $^{8}$, et, dans un registre différent mais sur une thématique proche, les différents cahiers du Carnet d'or ${ }^{9}$, partout les espaces se croisent et se répondent. II me semble que c'est dans les Mémoiresd'une survivantequel'image cit.

8. D. Lessing, Mémoires d'une survivante, op.

9. Id., Carnet d'or, Paris, Albin Michel, 1976 (éd. originale 1962). est la plus forte, lorsque l'héroïne se retrouve à accueillir une très jeune fille, Émilie, et que du fait même, semble-t-il, de cette responsabilité nouvelle qui lui incombe, s'ouvre "de l'autre côté » (du mur du salon) et de façon absolument aléatoire tout un ensemble de pièces qui débouchent elles-mêmes sur des scènes de l'enfance d'Émilie. Comme si l'adolescente nouvellement arrivée transportait avec elle l'ensemble de son passé qu'elle impose ainsi à l'adulte qui n'a pas d'autre choix que de s'y retrouver projetée. Comme si nous n'étions pas tous et toutes, au moment où les enfants doivent trouver leur place dans le monde, obligé-e-s de les accompagner avec tout ce qu'ils et elles ont reçu de leur enfance. Derrière et avec nous s'ouvrent des immensités et des dédales. Le talent littéraire permettant à Doris Lessing de mettre en scène cette problématique est alors double: d'une part, Émilie est accueillie par une adulte qui n'est pas sa mère, et peut ainsi regarder son passé avec sérénité puisqu'elle n'en est pas la cause, tout en y portant attention du fait du souci qu'elle a de l'adolescente ; d'autre part, Doris Lessing pose comme acquise, avec une étonnante audace, l'existence de ce "monde parallèle ", "derrière le mur » qui s'ouvre sans crier gare - et il faut quelques pages, aidées, pour ce qui me concerne, de la lecture antérieure d'autres ouvrages de l'auteure, pour accepter de « jouer le jeu ». On retrouve à cet égard dans l'étonnante fin des Enfants de la violence une interprétation tout à fait singulière de la folie comme ultra-lucidité dans un monde à la dérive.

Ainsi, tout au long d'une œuvre vaste et puissante, Doris Lessing montre que personne ne peut penser la vie d'autrui mais que penser sa propre vie est une tâche quotidienne, faisable à condition de ne pas s'obliger, dans un 
sursaut volontariste ou démesurément inquiet, à prendre en charge ce que l'on est en fait incapable d'assumer. L'assertion vaut notamment pour les enfants, dont Doris Lessing dit avec beaucoup de justesse dans son autobiographie $^{10}$ comment, pour ce qui concerne les siens, elle a confié les deux premiers à la garde d'autres personnes (le terme " abandonner", parfois employé, avec sa charge morale, me semble tout à fait impropre) tandis que "bébé Peter » a été un repère précieux pour elle lors de son arrivée en Angleterre. Le "je n'ai pas renoncé à comprendre le monde ", qui donne le titre de l'entretien déjà cité, peut alors s'interpréter en " je n'ai pas renoncé à mettre de l'ordre dans le chaos et à tenter de comprendre ce que je peux tenter d'assumer afin de faire un tant soit peu reculer la violence ». Comprendre la réalité, non pas pour la maîtriser mais pour tenter de s'y insérer au mieux. C'est une belle leçon.

Françoise Hatchuel ${ }^{*}$

10. D. Lessing, Dans ma peau, Paris, Albin Michel, 1995 (éd. originale 1994).

* Maîtresse de conférences en sciences de l'éducation. Auteure de Savoir apprendre transmettre. Une approche psychanalytique du rapport au savoir, Paris, La Découverte, coll. " La Découverte-poche », 2007. 